\title{
On a Conjecture of Alley and Alder for Fluids and Lorentz Models
}

\author{
M. H. Ernst ${ }^{1}$ and H. van Beijeren ${ }^{2}$
}

Received October 17, 1980

We discuss a conjecture of Alley and Alder predicting a relation between the four-point and the two-point velocity autocorrelation functions for fluids and Lorentz models at sufficiently long times. If the conjecture is correct a modified Burnett coefficient can be defined, which has a finite value, contrary to the ordinary Burnett coefficient, which is divergent. The conjecture is tested for four classes of models with different methods: for three-dimensional fluids modecoupling theory yields a negative result. The conjecture is confirmed for the $d$-dimensional deterministic Lorentz gas $(d \geqslant 2)$ and for a class of $d$-dimensional stochastic Lorentz models $(d \geqslant 1)$ by low-density kinetic theory, as well as by rigorous results, available for one dimension. For yet another class of onedimensional stochastic Lorentz models, which are exactly solvable in one dimension, the result is negative again. All four classes of models show long-time tails in the velocity autocorrelation function and have a finite diffusion coefficient.

KEY WORDS: Random walk on random lattice; waiting time (hopping) model; Lorentz models; hard sphere fluid; long-time tails; 2- and 4-point correlation function; Burnett coefficient.

\section{INTRODUCTION}

\subsection{Definitions}

In generalized hydrodynamics ${ }^{(1-4)}$ self-diffusion in equilibrium may be described by the equation

$$
\partial_{t} F(\mathbf{k}, t)=-k^{2} \int_{0}^{t} d \tau \phi(\mathbf{k}, \tau) F(\mathbf{k}, t-\tau)
$$

\footnotetext{
${ }^{1}$ Instituut voor Theoretische Fysica, Rijksuniversiteit, Utrecht, Princetonplein 5, P.O. Box 80006,3508 TA Utrecht, The Netherlands.

${ }^{2}$ Institut für Theoretische Physik, RWTH Aachen, Templergraben 55, 5100 Aachen, West Germany.
} 
or

$$
G(\mathbf{k}, z)=\left[z+k^{2} \hat{U}(\mathbf{k}, z)\right]^{-1}
$$

Here $F(\mathbf{k}, t)$ is the spatial Fourier transform of the probability density $P(\mathbf{r}, t)$ to find a test particle at position $\mathbf{r}$, with initial condition $P(\mathbf{r}, 0)$ $=\delta(\mathbf{r}) . G(\mathbf{k}, z)$ is the Laplace transform of $F(\mathbf{k}, t)$ and $\phi(\mathbf{k}, t)$ is the memory function. Its Laplace transform $\hat{U}(\mathbf{k}, z)$ is the generalized diffusion coefficient. Equation (1.1) may be viewed as a generalization of Fick's law. The phenomenological coefficient of self-diffusion $D$ is obtained from $\hat{U}(\mathbf{k}, z)$ as

$$
D=\lim _{z \rightarrow 0} \lim _{k \rightarrow 0} \hat{U}(\mathbf{k}, z)
$$

provided the limits exist. Furthermore, one may introduce the k-dependent velocity autocorrelation function

$$
C(\mathbf{k}, t)=\left\langle j_{\mathbf{k}}^{*}(0) j_{\mathbf{k}}(t)\right\rangle_{0}=\left\langle v_{x}(0) v_{x}(t) e^{-i k \Delta x(t)}\right\rangle_{0}
$$

where the $x$ axis is taken parallel to the $\mathbf{k}$ vector. The current $j_{\mathrm{k}}(t)=v_{x}(t)$ $e^{i k x(t)}$ and the displacement $\Delta x(t)=x(t)-x(0)$, where $x(t)$ and $v_{x}(t)$ are the $x$ components of position and velocity of the test particle at time $t$, and the asterisk denotes complex conjugation. The function $\hat{U}(\mathbf{k}, z)$ is connected to the Laplace transform of $C(\mathbf{k}, t)$ by the relation

$$
\hat{U}(\mathbf{k}, z)=z \hat{C}(\mathbf{k}, z) /\left[z-k^{2} \hat{C}(\mathbf{k}, z)\right]
$$

as derived in Ref. 3. By expanding $\phi(\mathbf{k}, t)$ in Eq. (1.1a) in powers of $k$ we obtain

$$
\partial_{t} F(\mathbf{k}, t)=\sum_{n=1}^{\infty}(-1)^{n} k^{2 n} \int_{0}^{t} d t^{\prime} \phi_{2 n}\left(t^{\prime}\right) F\left(\mathbf{k}, t-t^{\prime}\right)
$$

and we have similar $k$ expansions for

$$
\begin{aligned}
& \hat{U}(\mathbf{k}, z)=\hat{U}_{0}(z)-k^{2} \hat{U}_{2}(z)+\cdots \\
& \hat{C}(\mathbf{k}, z)=\hat{C}_{0}(z)-k^{2} \hat{C}_{2}(z)+\cdots
\end{aligned}
$$

Odd powers vanish for reasons of symmetry. The functions $\phi_{2 n}(t)$ or their Laplace transforms $\hat{U}_{2 n-2}(z)$ can be expressed in multitime velocity autocorrelation functions by expressing $\hat{U}$ in terms of $\hat{C}$ by means of (1.4), expanding $\hat{C}$ in powers of $k$ and using the relation $\Delta x(t)=\int_{0}^{t} v_{x}\left(t^{\prime}\right) d t^{\prime}$, so that

$$
\begin{aligned}
& \hat{U}_{0}(z)=\hat{C}_{0}(z) \\
& \hat{U}_{2}(z)=\hat{C}_{2}(z)-(1 / z) \hat{C}_{0}^{2}(z)
\end{aligned}
$$


and in time language

$$
\begin{aligned}
& \phi_{2}(t)=C_{0}(t)=\left\langle v_{x}(0) v_{x}(t)\right\rangle_{0} \\
& \phi_{4}(t)=C_{2}(t)-\int_{0}^{t} d \tau \int_{0}^{\tau} d \tau^{\prime} C_{0}\left(\tau^{\prime}\right) C_{0}\left(\tau-\tau^{\prime}\right)
\end{aligned}
$$

where

$$
C_{2}(t)=\int_{0}^{t} d \tau \int_{0}^{\tau} d \tau^{\prime}\left\langle v_{x}(0) v_{x}\left(\tau^{\prime}\right) v_{x}(\tau) v_{x}(t)\right\rangle_{0}
$$

Equation (1.5) can also be brought into a form resembling the ordinary form of Fick's law, ${ }^{(3)}$ i.e.,

$$
\partial_{t} F(\mathbf{k}, t)=\left[-k^{2} D(t)+k^{4} B(t)+\cdots\right] F(\mathbf{k}, t)
$$

with

$$
\begin{aligned}
B(t) & =D_{4}(t)-\int_{0}^{t} d t^{\prime}\left[D(t)-D\left(t^{\prime}\right)\right] D\left(t-t^{\prime}\right) \\
D(t) & =\int_{0}^{t} d t^{\prime} \phi_{2}\left(t^{\prime}\right) \\
D_{4}(t) & =\int_{0}^{t} d t^{\prime} \phi_{4}\left(t^{\prime}\right)
\end{aligned}
$$

This can be seen most easily by making in Eq. (1.5) the replacement

$$
F\left(\mathbf{k}, t-t^{\prime}\right)=F(\mathbf{k}, t)+\int_{0}^{t^{\prime}} d \tau \partial_{\tau} F(\mathbf{k}, t-\tau)
$$

followed by iteration and expansion in powers of $k$. If the correlation functions $\phi_{2 n}(t)$ decay sufficiently fast for long times, $D(t)$ and $B(t)$ will approach, respectively, the ordinary diffusion coefficient $D$ and the ordinary super Burnett coefficient $B$, provided they exist. However, the correlation functions $\phi_{2 n}(t)$ decay in general very slowly, ${ }^{(4-9)}$ proportional to $t^{-\beta}$, where $\beta$ depends on the models and on the number of dimensions considered, and the coefficients $D(t), B(t)$ or higher-order ones can be shown to diverge for long times, in a manner depending on the values of $\beta$.

\subsection{Models}

We will consider the long-time behavior of the quantities discussed above for four different classes of models.

The first class consists of three-dimensional one-component fluids with short-range spherically symmetric pair interactions, for which we quote the existing mode-coupling results. ${ }^{(3,10)}$ In the same class of models molecular dynamics results are reported for the special case of hard spheres. ${ }^{(5,11,12)}$ 
The second class of models consists of $d$-dimensional $(d \geqslant 2)$ deterministic Lorentz models in two or more dimensions, which is the standard Lorentz gas. ${ }^{(8,13)}$ This system consists of fixed spherical scatterers of radius $\sigma$, randomly distributed in space according to a certain probability distribution. In addition there is a light point particle, which moves at constant speed and is reflected specularly upon collision with a scatterer. These models are studied by means of low-density kinetic theory, following the method of Ernst and Weyland. ${ }^{(7)}$ Molecular dynamics results for these models are discussed in Refs. 14-18.

The third class of models are $d$-dimensional $(d \geqslant 1)$ stochastic Lorentz models. ${ }^{(19,20)}$ These models are similar to the deterministic Lorentz models, but the reflection law describing the collision of a light particle with a scatterer is of a stochastic nature-i.e., if the light particle with velocity $v \hat{v}$ and position $\mathbf{r}$ hits a scatterer centered at position $\mathbf{R}$, it is reflected with velocity $v \hat{\mathbf{v}}^{\prime}$ with a probability described by a kernel $K\left(\hat{\mathbf{v}}^{\prime}, \hat{\mathbf{v}}, \hat{\boldsymbol{\sigma}}\right)$, where $\hat{\boldsymbol{\sigma}}=(\mathbf{r}-\mathbf{R}) /|\mathbf{r}-\mathbf{R}|$ and $\hat{\mathbf{a}}$ is a unit vector in the direction of $\mathbf{a}$. A special case is a diffuse reflection law, where the outgoing velocity is completely independent of the incident one, i.e.,

$$
K_{\mathrm{diff}}\left(\hat{\mathbf{v}}^{\prime}, \hat{\mathbf{v}}, \hat{\boldsymbol{\sigma}}\right)=\frac{\left(\hat{\mathbf{v}}^{\prime} \cdot \hat{\boldsymbol{\sigma}}\right) \theta\left(\hat{\mathbf{v}}^{\prime} \cdot \hat{\boldsymbol{\sigma}}\right)}{\int d \hat{\mathbf{v}}^{\prime}\left(\hat{\mathbf{v}}^{\prime} \cdot \hat{\boldsymbol{\sigma}}\right) \theta\left(\hat{\mathbf{v}}^{\prime} \cdot \hat{\boldsymbol{\sigma}}\right)}
$$

Here $\theta(x)$ is the unit step function. Actually, deterministic Lorentz models may also be considered as a special case with

$$
K_{\text {spec }}\left(\hat{\mathbf{v}}^{\prime}, \hat{\mathbf{v}}, \hat{\boldsymbol{\sigma}}\right)=\delta\left(\hat{\mathbf{v}}-2 \hat{\boldsymbol{\sigma}}(\hat{\boldsymbol{\sigma}} \cdot \hat{\mathbf{v}})-\hat{\mathbf{v}}^{\prime}\right)
$$

In the one-dimensional case stochastic Lorentz models have nontrivial dynamics, whereas in deterministic Lorentz models the light particle simply keeps running back and forth between two neighboring scatterers. ${ }^{(2))}$ The low-density kinetic theory methods apply equally well to the deterministic and stochastic Lorentz models. ${ }^{(7,22)}$ In addition some rigorous results are available in the one-dimensional case, ${ }^{(19,20)}$ and molecular dynamics data have been published for two-dimensional ${ }^{(14-18)}$ and one-dimensional systems. ${ }^{(22)}$

The fourth class of models to be considered are waiting-time Lorentz models ${ }^{(19)}$ in one dimension. In these models the scatterers are distributed along a line according to a given probability distribution; the light particle does not move at constant speed, but sits instead on one of the scatterers and jumps instantaneously to a neighboring scatterer after a stochastically distributed waiting time. The concept "neighbor" is well defined in one dimension; in higher dimensions it should be specified more precisely. These models are very similar to ordinary random walks with a waiting time distribution. ${ }^{(23)}$ An essential difference however, responsible for long- 
time tails, arises from the stochastic spatial distributions of the scatterers. A large subclass of waiting-time Lorentz models can be solved exactly in one dimension, ${ }^{(19)}$ by virtue of their close resemblance to ordinary random walks.

\subsection{Long-Time Behavior}

After this introduction of models we briefly review the long-time behavior of some of their time-correlation functions. In the threedimensional fluid one has

$$
\phi_{2}(t) \sim t^{-3 / 2}, \quad B(t) \sim t^{1 / 2}
$$

The result for $\phi_{2}(t)$ was obtained in Refs. 5, 6, and 9 and for $B(t)$ in Refs. 3 and 10 .

For the $d$-dimensional deterministic Lorentz gas $(d \geqslant 2)$ and for the $d$-dimensional stochastic Lorentz gas $(d \geqslant 1)$ one has ${ }^{(7,19,20,22)}$

$$
\begin{array}{ll}
\phi_{2}(t) \sim t^{-1-d / 2} & \\
B(t) \sim \log t & (d=2) \\
B(t) \sim t^{1 / 2} & (d=1)
\end{array}
$$

The result for $B(t)$ is new, and will be derived here from low-density kinetic theory.

For the one-dimensional waiting-time Lorentz models one has ${ }^{(19)}$

$$
\begin{gathered}
\phi_{2}(t) \sim t^{-3 / 2} \\
B(t) \sim t^{1 / 2}
\end{gathered}
$$

as will be briefly derived here.

\subsection{Conjecture of Alley and Alder}

The purpose of this paper is to test an interesting conjecture made by Alley and Alder, ${ }^{(16-18)}$ based on evidence from molecular dynamics experiments. For systems in which the diffusion coefficient $D$ exists, these authors conjecture "that the four-point correlation function involved in $\phi_{4}$ decays at large times sufficiently fast that it can be expressed in terms of the two-point function $\phi_{2}$." More specifically, the conjecture implies that in Lorentz models at long times $\phi_{4}(t)$ should be proportional to $\phi_{2}(t)$, whereas a more speculative conjecture for the three-dimensional fluid implies that there should exist a similar proportionality between $\phi_{2}(t)$ and $\phi_{4 h}(t)$, defined as

$$
\phi_{4 h}(t)=\phi_{4}(t)-D^{\prime} t \phi_{2}(t)
$$


where $D^{\prime}=D \bar{\nu} /(D+\bar{\nu})$ and $\bar{\nu}$ is the kinematic viscosity. In fact Alley and Alder extend this conjecture to one involving $\phi_{2 n}(t)$ for general values of $n$. For the Lorentz gas this extension implies that $\phi_{2 n}(t) \sim \phi_{2}(t)$ for large $t$ and all $n \geqslant 2$; for the three-dimensional fluid a similar property is proposed for a set of functions $\phi_{2 n h}(t)$. Unfortunately, the definitions of $\phi_{2 n h}$ for $n>2$ are not clearly specified. For the time being we restrict our discussion to the case $n=2$.

An implication of the conjecture of Alley and Alder is that a modified Burnett coefficient exists, which reads for Lorentz models

$$
D_{4}=\lim _{t \rightarrow \infty} D_{4}(t)=\lim _{t \rightarrow \infty} \int_{0}^{t} d t^{\prime} \phi_{4}\left(t^{\prime}\right)<\infty
$$

and for three-dimensional fluids

$$
D_{4 h}=\lim _{t \rightarrow \infty} D_{4 h}(t)=\lim _{t \rightarrow \infty} \int_{0}^{t} d t^{\prime} \phi_{4 h}\left(t^{\prime}\right)<\infty
$$

We will investigate the conjecture for the four classes of models, discussed in Section 1.2, using the methods as specified before. Since arguments based on a rather complicated low-density kinetic theory may not be completely convincing, and since a phenomenological theory such as modecoupling theory ${ }^{3}$ could possibly predict the $\phi_{2}(t)$ tails correctly but predict the $\phi_{4}(t)$ tails incorrectly, it is important to check the conjecture against rigorous results.

The plan of the paper is as follows. In Section 2 we discuss the fluid, in Section 3 deterministic and stochastic Lorentz models, and in Section 4 the one-dimensional waiting-time Lorentz model. A discussion of the results and a comparison with molecular dynamics results is presented in Section 5 .

\section{THREE-DIMENSIONAL FLUID}

For a fluid of particles with mass $m$ in thermal equilibrium at a temperature $T$ with an arbitrary density $n$, all necessary correlation functions have already been calculated by means of mode-coupling theory. The required results can be obtained from Refs. 3 and 10, and the conjecture can be tested directly.

We quote from the literature the long-time behavior of the velocity

\footnotetext{
${ }^{3}$ A phenomenological theory for the long-time effects in a $d$-dimensional Lorentz gas at general densities has been developed recently by Dorfman, Ernst, and van Beijeren (to be published). It reduces in the low-density limit to known results for the tail of the velocity correlation function; it predicts correctly the exactly known coefficients of the tails in the one-dimensional stochastic Lorentz models; and it confirms the results for the Burnett coefficients obtained here.
} 
correlation function [Eq. (2.12b) of Ref. 3]

$$
\phi_{2}(t)=C_{0}(t) \simeq \frac{k_{B} T}{12 \pi^{3 / 2} m n(D+\bar{\nu})^{3 / 2} t^{3 / 2}}
$$

and of the Burnett correlation function ${ }^{4}$ [Eq. (2.13b) of Ref. 3]

$$
\phi_{4}(t)=U_{2}(t) \simeq \frac{k_{B} T D(5 \bar{\nu}+2 D)}{60 \pi^{3 / 2} m n(D+\bar{v})^{5 / 2}} \frac{1}{t^{1 / 2}}
$$

These results have also been obtained from a low-density kinetic theory. ${ }^{(3)}$ The correlation function $\phi_{4 h}(t)$ of Alley and Alder, defined in (1.16), behaves now as

$$
\phi_{4 h}(t) \simeq \frac{k_{B} T D^{2}}{30 \pi^{3 / 2} m n(D+\bar{\nu})^{5 / 2}} \frac{1}{t^{1 / 2}}
$$

and the modified Burnett coefficient $D_{4 h}(t)$ diverges for large times $\sim t^{1 / 2}$. We can, therefore, conclude that for the three-dimensional fluid the conjecture is in conflict with mode-coupling theory, as well as low-density kinetic theory - the very theories that have been so successful in explaining the long-time tails themselves.

In view of this conclusion let us consider the molecular dynamics evidence upon which the conjecture of Alley and Alder is based, i.e., the calculations by Wood of $\phi_{2}(t)$ and $D_{4}(t)$, as represented in Figs. (5.2) and (5.5) of Ref. 11. In Fig. 1, made available to us by Wood, $\Psi(s)=\alpha d B(t) / d t$ is plotted as a function of the dimensionless time $s=v t$, when $\nu^{-1}$ is the actual (Enskog) mean free time between collisions, and the normalization constant $\alpha=\left(2 D_{0}\right)^{-2}=(16 / 9) \pi \beta m n^{2} \sigma^{4}$, where $D_{0}$ is the Boltzmann value for the hard sphere diffusion coefficient and $\sigma$ the diameter of the sphere. Furthermore we find from (1.10)

$$
\frac{d B(t)}{d t}=\phi_{4}(t)-\frac{d}{d t} \int_{0}^{t} d t^{\prime}\left[D(t)-D\left(t^{\prime}\right)\right] D\left(t-t^{\prime}\right)
$$

Asymptotically the second term on the right-hand side of (2.4) behaves as - Dt $\phi_{2}(t)$, as can be deduced from (1.10) and (2.1), and on using (1.16) we obtain from (2.4)

$$
\frac{d B(t)}{d t} \sim \phi_{4 h}(t)-\frac{D^{2} t}{D+\bar{\nu}} \phi_{2}(t)
$$

According to Alley and Alder's conjecture one has asymptotically ${ }^{(16-18)}$

$$
\phi_{4 h}(t) \sim \frac{D_{4 h}}{D_{h}} \phi_{2}(t)=\frac{D_{4 h}(D+\bar{v})}{D^{2}} \phi_{2}(t)
$$

\footnotetext{
${ }^{4}$ Equation (2.13b) of Ref. 3 contains a typographical error, viz., $\pi$ should be replaced by $\pi^{3 / 2}$.
} 


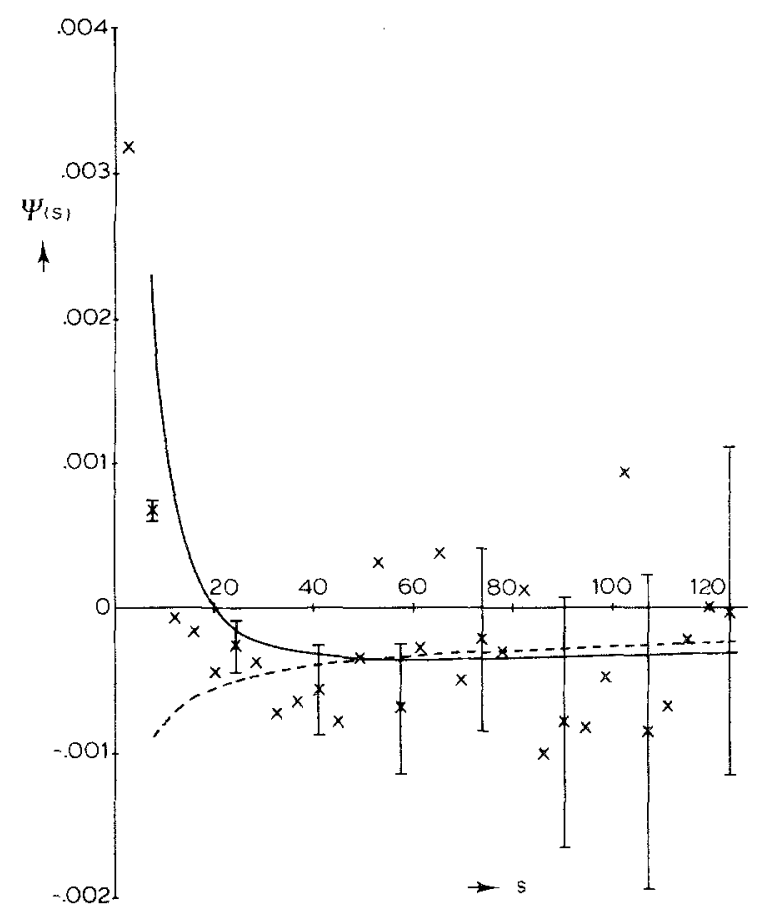

Fig. 1. The correlation function $\Psi(s)=\alpha d B(t) / d t$, defined in (2.4), as a function of the dimensionless time $s=\nu t$.

where $D_{4 h}$ has been determined in Refs. $16-18$ as $D_{4 h} \simeq 1.68 D_{E}^{2} / \nu$ and $D_{E}$ is the Enskog value of the hard sphere diffusion coefficient.

Combining (2.4)-(2.6) leads to the prediction of the conjecture, as represented by the solid line in Fig. 1. Insertion of (2.3) into (2.5) leads to the prediction of the mode-coupling theory, as represented by the dashed line in Fig. 1. Both curves are consistent with the molecular dynamics data, as can be seen in Fig. 1.

\section{DETERMINISTIC AND STOCHASTIC LORENTZ MODELS}

\subsection{Low-Density Kinetic Theory}

We will calculate the long-time behavior of the correlation functions $\phi_{2}(t), \phi_{4}(t), D(t)$, and $B(t)$ from low-density kinetic theory. Because of the restriction to low densities our results will not depend on short-range spatial correlations between scatterers, such as overlap exclusions.

We need a few concepts from kinetic theory..$^{(3,7,8)}$ The Laplace transform $\hat{C}(\mathbf{k}, z)$ of the velocity autocorrelation function, defined in Eq. (1.3), 
can be expressed in terms of an exact propagator $\Gamma_{\mathbf{k}}(z)$, i.e.,

$$
\hat{C}(\mathbf{k}, z)=v^{2}\left\langle\hat{\boldsymbol{v}}_{x} \boldsymbol{\Gamma}_{\mathbf{k}}(z) \hat{\boldsymbol{v}}_{x}\right\rangle
$$

where the average without a subscript $\langle\cdots\rangle=\left(\int d \hat{v} \cdots\right) / \Omega_{d}$ is an average over a $d$-dimensional unit sphere of surface area $\Omega_{d}=2 \pi^{d / 2} / \Gamma(d / 2)$. In the low-density limit $\mathbf{\Gamma}_{\mathbf{k}}(z)$ reduces to the Boltzmann propagator

$$
\Gamma_{\mathbf{k}}^{B}(z)=\left(z-i \mathbf{k} \cdot \mathbf{v}-n \mathbf{T}_{0}\right)^{-1}
$$

with a Boltzmann collision operator defined as

$$
n \mathbf{T}_{0} f(\hat{\mathbf{v}})=n v \boldsymbol{\sigma}^{d-1} \int^{\prime} d \hat{\boldsymbol{\sigma}} \int^{\prime \prime} d \hat{\mathbf{v}}^{\prime}|\hat{\mathbf{v}} \cdot \hat{\boldsymbol{\sigma}}| K\left(\hat{\mathbf{v}}^{\prime}, \hat{\mathbf{v}}, \hat{\boldsymbol{\sigma}}\right)\left[f\left(\hat{\mathbf{v}}^{\prime}\right)-f(\hat{\mathbf{v}})\right]
$$

where $n$ is the density of scatterers. The $\hat{\boldsymbol{\sigma}}$ integration is restricted to the hemisphere $\hat{\mathbf{v}} \cdot \hat{\boldsymbol{\sigma}}<0$ and the $\hat{\mathbf{v}}^{\prime}$ integration to the hemisphere $\hat{\mathbf{v}}^{\prime} \cdot \hat{\boldsymbol{\sigma}}>0$. Furthermore, the kernel $K$ obeys the obvious normalization $\int d \hat{\mathbf{v}}^{\prime} K\left(\hat{\mathbf{v}}^{\prime}, \hat{\mathbf{v}}, \hat{\boldsymbol{\sigma}}\right)$ $=1$, as can be seen in the two examples (1.12). The low-density diffusion coefficient $D$ can be obtained from (1.10) and (3.1)-(3.3) as

$$
D=-\frac{v^{2}}{d n\left\langle\hat{\mathbf{v}} \cdot \mathbf{T}_{0} \hat{\mathbf{v}}\right\rangle}
$$

Of course, its precise value depends on the kernel $K$, e.g., for diffuse reflection one obtains from (1.12a)

$$
D=\left(v^{2} / d \nu\right)\left[1+\frac{1}{4} B^{2}\left(\frac{1}{2}, \frac{1}{2}(d+1)\right)\right]^{-1}
$$

and for specular reflection from $(1.12 b)$

$$
D=\left(v^{2} / \nu\right)(d+1) / 4 d
$$

Here $\nu$ is the collision frequency, given in both cases as

$$
\nu=n v \sigma^{d-1} \int^{\prime} d \hat{\sigma}|\hat{\mathbf{v}} \cdot \hat{\boldsymbol{\sigma}}|=\frac{n v \sigma^{d-1} \pi^{(d-1) / 2}}{\Gamma\left[\frac{1}{2}(d+1)\right]}
$$

and $B(x, y)=\Gamma(x) \Gamma(y) / \Gamma(x+y)$.

We are mainly interested in the small- $k$ and $-z$ behavior of the kinetic propagator, which for low densities can be represented as

$$
\Gamma_{\mathbf{k}}^{B}(z) \simeq \Gamma_{\mathbf{k}}^{s}(z)+\Gamma_{\mathbf{k}}^{f}(z)
$$

with

$$
\begin{aligned}
& \boldsymbol{\Gamma}_{\mathbf{k}}^{s}(z) \simeq\left(z+k^{2} D\right)^{-1} P_{\mathbf{k}} \\
& \boldsymbol{\Gamma}_{\mathbf{k}}^{f}(z) \simeq-Q_{\mathbf{k}}\left(i \mathbf{k} \cdot \mathbf{v}+n \mathbf{T}_{0}\right)^{-1} Q_{\mathbf{k}}
\end{aligned}
$$

Hence $\Gamma^{B}$ is separated into a slowly decaying hydrodynamic part $\Gamma^{s}$ and a fast decaying part $\boldsymbol{\Gamma}^{f}$. The operator $P_{\mathbf{k}}=1-Q_{\mathbf{k}}$ projects on the hydrodynamic mode $\psi_{\mathbf{k}}(\hat{\mathbf{v}})$ of the Boltzmann propagator, i.e., $P_{\mathbf{k}} f(\hat{\mathbf{v}})=\psi_{\mathbf{k}}(\hat{\mathrm{v}})\left\langle\psi_{\mathbf{k}}(\hat{\mathbf{v}})\right.$ 
$f(\hat{v})\rangle$. (Note the absence of complex conjugation inside the average.) The hydrodynamic mode satisfies

$$
\left(i \mathbf{k} \cdot \mathbf{v}+n \mathbf{T}_{0}\right) \psi_{\mathbf{k}}(\hat{\mathbf{v}}) \simeq\left\{-k^{2} D+O\left(k^{4}\right)\right\} \psi_{\mathbf{k}}(\hat{\mathbf{v}})
$$

and is easily found to be $\psi_{\mathbf{k}} \simeq 1+i \mathbf{k} \cdot \hat{\mathbf{v}}(d D / v)+O\left(k^{2}\right)$.

\subsection{Ring Events}

The velocity autocorrelation function in the low-density region has a long-time tail, ${ }^{(7)}$ which is expected to result from single ring events. These events consist of a sequence of collisions of the light particle with a scatterer, separated by free streaming, such that precisely two collisions occur with the same scatterer. Schematically, the sum of the contributions from all these events to the velocity autocorrelation function may be represented by $n\left\langle v_{x} \Gamma^{B} \mathbf{T}(1) \Gamma^{B} \mathbf{T}(1) \Gamma^{B} v_{x}\right\rangle$, and its asymptotic behavior reads

$$
\phi_{2}(t) \simeq-\frac{2 \pi D^{2}}{n}\left(\frac{1}{4 \pi D t}\right)^{1+d / 2}
$$

as may be verified with the methods of the Appendix. Similarly, $D(t)$ defined in (1.10) has a long-time behavior

$$
D(t) \simeq D\left[1+\frac{1}{n d}\left(\frac{1}{4 \pi D t}\right)^{d / 2}\right]
$$

For the calculation of $\phi_{4}(t)$, defined in Eq. (1.7b), we consider first the four-point correlation function (1.8) and use the low-density kinetic theory of Ref. [3]. The dominant small- $z$ singularities are expected to arise from diagrams of classes I and V, defined in Ref. 3. In the schematic notation, introduced above, the dominant low-density contributions to each of these classes of diagrams have the structure

$$
\begin{aligned}
& \hat{C}_{2}^{(\mathrm{I})}(z)=n\left\langle v_{x} \boldsymbol{\Gamma}^{B}\left[\mathbf{T}(1) \Gamma^{B} v_{x} \Gamma^{B} v_{x} \Gamma^{B} \mathbf{T}(1)\right] \boldsymbol{\Gamma}^{B} v_{x}\right\rangle \\
& \hat{C}_{2}^{(\mathrm{II})}(z)=n^{2}\left\langle v_{x} \boldsymbol{\Gamma}^{B}\left[\mathbf{T}(1) \boldsymbol{\Gamma}^{B} v_{x} \boldsymbol{\Gamma}^{B} \mathbf{T}(1)\right]\right. \\
& \left.\times \boldsymbol{\Gamma}^{B}\left[\mathbf{T}(2) \boldsymbol{\Gamma}^{B} v_{x} \Gamma^{B} \mathbf{T}(2)\right] \boldsymbol{\Gamma}^{B} v_{x}\right\rangle \\
& \hat{C}_{2}^{(I I I)}(2)=n\left\langle v_{x} \Gamma^{B}\left[\mathbf{T}(1) \Gamma^{B} v_{x} \Gamma^{B} \mathbf{T}(1)\right] \boldsymbol{\Gamma}^{B} v_{x} \Gamma^{B} v_{x}\right\rangle \\
& \hat{C}_{2}^{(\mathrm{IV})}(z)=n\left\langle v_{x} \boldsymbol{\Gamma}^{B} v_{x} \Gamma^{B}\left[\mathbf{T}(1) \boldsymbol{\Gamma}^{B} v_{x} \boldsymbol{\Gamma}^{B} \mathbf{T}(1)\right] \boldsymbol{\Gamma}^{B} v_{x}\right\rangle \\
& \hat{C}_{2}^{(\mathrm{V})}(z)=\left\langle v_{x} \Gamma^{B} v_{x} \Gamma^{B} v_{x} \Gamma^{B} v_{x}\right\rangle
\end{aligned}
$$

\subsection{Confirmation of Conjecture}

The line of argument will be that for the quantities defined in (1.7)(1.10), the leading small- $z$ singularity in $\hat{U}_{2}(z)$ is the same as in $\hat{C}_{0}(z)$, or 
that for the inverse Laplace transforms the dominant long-time tail in $\phi_{4}(t)$ is the same as in $\phi_{2}(t)$. Hence the diffusion coefficient $D$, as well as the modified Burnett coefficient, $D_{4}$, exist, thus confirming the conjecture.

We start our calculations by considering first the contributions of the class- $\mathrm{V}$ diagrams, i.e.,

$$
\begin{aligned}
\hat{C}_{2}^{(\mathrm{V})}(z) & =v^{4}\left\langle\hat{v}_{x} \boldsymbol{\Gamma}_{0}(z) \hat{v}_{x} \boldsymbol{\Gamma}_{0}(z) \hat{v}_{x} \boldsymbol{\Gamma}_{0}(z) \hat{v}_{x}\right\rangle \\
& =\frac{2(d-1)}{d+2} \hat{\gamma}_{2}(z) \hat{C}_{0}^{2}(z)+\frac{1}{z} \hat{C}_{0}^{2}(z)
\end{aligned}
$$

Here the exact propagator $\Gamma_{0}(z)$, is an isotropic operator acting on functions of $\hat{v}$. Its eigenfunctions are the (hyper) spherical harmonics $1, \hat{v}$, $\{\hat{v} \hat{v}\}_{s}=\hat{v} \hat{v}-(1 / d) 1, \ldots$, where $\mathbb{1}$ is a unit tensor. Its eigenvalues $\hat{\gamma}_{l}(z)$ are, respectively,

$$
\begin{aligned}
& \hat{\gamma}_{0}(z)=z^{-1} \\
& \hat{\gamma}_{1}(z)=\left\langle\hat{\mathbf{v}} \cdot \boldsymbol{\Gamma}_{0}(z) \hat{\mathbf{v}}\right\rangle=d \hat{C}_{0}(z) / v^{2} \\
& \hat{\gamma}_{2}(z)=\frac{d}{d-1}\left\langle\{\hat{\mathbf{v}} \hat{\mathbf{v}}\}_{s}: \Gamma_{0}(z)\{\hat{\mathbf{v}} \hat{\mathbf{v}}\}_{s}\right\rangle
\end{aligned}
$$

In the low-density and long-time limit the inverse Laplace transforms $\gamma_{l}(t)$ behave like $\gamma_{l}(t) \simeq t^{-d / 2-l}$. For $l=1$ we have the tail of the velocity correlation function; the result for larger $l$ is a straightforward extension of the method of Ref. 7. The projection of the hydrodynamic mode $\psi_{k}(\hat{v})$ onto the $l$ th-order spherical harmonic is proportional to $k^{l}$. The second line of Eq. (3.12) follows directly from the eigenvalues and eigenfunctions of $\Gamma_{0}(z)$. We also observe that $\hat{C}_{0}^{2} / z$ is canceled exactly by the subtracted term in $\hat{U}_{2}(z)$ or $\phi_{4}(t)$ in Eq. (1.7). The remaining contribution to $\hat{U}_{2}(z)$ has the same dominant singularity as $\hat{C}_{0}(z)$; its contribution to $\phi_{4}(t)$ is proportional to $\phi_{2}(t)$ in the long-time limit, and does not contribute to $D_{4}(t)$ a term of $O(\log t)$ for $d=2$ or of $O\left(t^{1-d / 2}\right)$ for $d \neq 2$. Since we are at present only interested in the leading low-density behavior of $\phi_{4}(t)$ we should neglect the difference in position of a scatterer and a light particle, when calculating the contribution from the diagrams of class I. This implies that in a Fourier representation of the quantity between parentheses in (3.11a) the binary collision operators $\mathbf{T}_{\mathbf{k}}$ and $\mathbf{T}_{-\mathbf{k}}$ may be replaced by $\mathbf{T}_{0} \cdot{ }^{(7,3)}$ Then the contribution from the class-I diagrams is obtained as

$$
\begin{aligned}
\hat{C}_{2}^{(\mathrm{I})}(z) & =v^{4} n\left\langle\hat{v}_{x} \boldsymbol{\Gamma}_{0}(z) \mathbf{T}_{0} B_{x x}(z) \mathbf{T}_{0} \boldsymbol{\Gamma}_{0}(z) \hat{v}_{x}\right\rangle \\
& =\frac{v^{4}}{n}\left[\frac{\hat{C}_{0}(z)}{D}\right]^{2}\left\langle\hat{v}_{x} B_{x x}(z) \hat{v}_{x}\right\rangle
\end{aligned}
$$

To lowest order in the density the operator $B_{x x}(z)$ is

$$
B_{x x}(z)=\int^{\prime} \frac{d \mathbf{k}}{(2 \pi)^{d}} \boldsymbol{\Gamma}_{\mathbf{k}}^{B}(z) \hat{v}_{x} \boldsymbol{\Gamma}_{\mathbf{k}}^{B}(z) \hat{v}_{x} \boldsymbol{\Gamma}_{\mathbf{k}}^{B}(z)
$$


The prime indicates that the integration over wave numbers $\mathbf{k}$ is restricted to $|\mathbf{k}| \lesssim k_{0}$, which is on the order of the inverse mean free path. In deriving the second equality in (3.14) we have used that the spherical harmonics are eigenfunctions of the isotropic operators $\boldsymbol{\Gamma}_{0}(z)$ and $\mathbf{T}_{0}$ with $\mathbf{T}_{0} \hat{v}=$ $-\hat{v}\left(v^{2} / d n D\right)$. To evaluate $\left\langle\hat{v}_{x} B_{x x}(z) \hat{v}_{x}\right\rangle$ we separate the Boltzmann propagators into their slow $(s)$ and fast $(f)$ part as defined in (3.7). The following combinations of slow and fast parts $\{(s s s),(s s f),(s f s),(f s s),(f s f)\}$. all contribute singular terms for small $z$, which behave as $z^{(d-2) / 2} \log z$ for even $d$, and as $z^{(d-2) / 2}$ for odd $d$. However, the sum of the coefficients of the leading singularity vanishes. The details of the calculations can be found in the Appendix. Therefore, the dominant singularity in $\hat{C}_{2}^{(I)}(z)$ is the same as in $\hat{C}_{0}(z)$, in agreement with the conjecture. It should be stressed that in the kinetic theory for a three-dimensional hard sphere fluid the contribution $\hat{C}_{2}^{(\mathrm{I})}(z)$ has a dominant singularity proportional to $1 / z^{1 / 2}$ with a nonvanishing coefficient, leading to the results of Section $2 .^{(3)}$

The contributions from the diagrams II, III, and IV can be analyzed in much the same way. They do not contribute to the dominant small- $z$ singularity. Inserting our results for $\hat{C}_{2}^{(\mathrm{I})}(z), \ldots, \hat{C}_{2}^{(\mathrm{V})}(z)$ into $(1.7 \mathrm{a})$ we see that $\hat{U}_{2}(z)$ has the same small-z singularity as $\hat{C}_{0}(z)$, so that $\phi_{4}(t)$ is proportional to $\phi_{2}(t)$ in the long-time limit.

In conclusion, we have confirmed in the low-density limit the conjecture of Alley and Alder, stating that $\phi_{4}(t) \sim \phi_{2}(t)$ for long times, for $d$-dimensional deterministic Lorentz models with $d \geqslant 2$, and stochastic Lorentz models with $d \geqslant 1$. This implies the existence of the modified Burnett coefficient $D_{4}$ in the same limit.

For a class of one-dimensional stochastic Lorentz models, as discussed in Section 1.2, van Beijeren and Spohn ${ }^{(19,20)}$ have shown rigorously that for small $z$ the functions $\hat{U}_{2}(z)$ and $\hat{U}_{0}(z)$, as defined in (1.6), both have a leading singularity proportional to $z^{1 / 2}$. Hence, the modified Burnett coefficient exists for this model.

\subsection{Ordinary Burnett Coefficient}

From Eqs. (1.10) and (3.10) we can deduce the low-density behavior of the ordinary Burnett coefficient,

$$
\begin{aligned}
& B(t) \simeq \frac{D^{2} t}{n}\left(\frac{1}{4 \pi D t}\right)^{1 / 2} \quad(d=1) \\
& B(t) \simeq \frac{D}{8 \pi n} \log (\nu t) \quad(d=2) \\
& B(t) \simeq B-\frac{D^{2} t}{(d-2) n}\left(\frac{1}{4 \pi D t}\right)^{d / 2}
\end{aligned}
$$


where $B$ is some constant. For $d=1,2$ the Burnett coefficient $B(t)$ shows the expected long-time divergence.

For a comparison with numerical data it is also convenient to give the Boltzmann values of the ordinary and modified Burnett coefficients for the deterministic Lorentz gas in $d$ dimensions, i.e.,

$$
\begin{aligned}
& D_{4}=\frac{(d-1)(d+3)}{d+2} \frac{D^{3}}{v^{2}} \\
& B=-\frac{3}{d+2} \frac{D^{3}}{v^{2}}
\end{aligned}
$$

where $D_{4}$ is directly given by the limit as $z \rightarrow 0$ of the first term in the second equality of (3.12), and $\hat{\gamma}_{2}(0)$, in the low-density limit, follows from (3.13) and (3.2). $B$ is calculated similarly from Eq. (1.10) and the lowdensity limit of $C_{0}(t)$ in (3.13).

\section{WAITING-TIME LORENTZ MODEL}

\subsection{Distributions for Interval Lengths and Waiting Times}

We consider another one-dimensional stochastic Lorentz model, characterized by a waiting-time distribution, which is symmetric for jumps in the right or left direction, independent of the scatterer on which the light particle is located, independent of the previous history, and given by $\frac{1}{2} \tilde{\psi}(t)$, with Laplace transform $\frac{1}{2} \psi(z)$. We refer to it briefly as the waiting-time Lorentz model.

The scatterers are labeled $(\ldots-1,0,1,2, \ldots)$ in sequence of their positions on the real axis, and it is assumed that the lengths of the intervals between neighboring scatterers are independent variables with a probability density $\mu(x)$, satisfying

$$
\begin{aligned}
\int_{0}^{\infty} d x \mu(x) & =\langle 1\rangle=1 \\
\int_{0}^{\infty} d x x \mu(x) & =\langle x\rangle=l \\
\int_{0}^{\infty} d x(x-l)^{2} \mu(x) & =\left\langle x^{2}\right\rangle-\langle x\rangle^{2}=\Delta^{2}
\end{aligned}
$$

This model is a special case of a class of more general one-dimensional waiting-time Lorentz models, introduced in Ref. 19, for which the Green's function $G(k, z)$ of Eq. (1.1) was calculated explicitly. Here we want to repeat this calculation for our special case insofar as it is needed to test the conjecture. 
The probability density $P(x, t)$ for the light particle to be at $x$ at time $t$ is

$$
P(x, t)=\sum_{n=-\infty}^{\infty} \tilde{P}_{n}(t) \mu_{n}(x)
$$

Here $\mu_{n}(x)$ is the probability density of finding the $n$th scatterer at $x$, with $\mu_{0}(x)=\delta(x)$, and $\tilde{P}_{n}(t)$ is the probability that the light particle sits on the $n$th scatterer at time $t$. The Fourier-Laplace transform of $P(x, t)$ can be expressed as

$$
\begin{aligned}
G(k, z) & =\int_{-\infty}^{\infty} d x \int_{0}^{\infty} d t e^{-i k x} e^{-z t} P(x, t) \\
& =P_{0}(z)+\sum_{n=1}^{\infty}\left[P_{n}(z) M^{n}(k)+P_{-n}(z) M^{n}(-k)\right]
\end{aligned}
$$

with

$$
M(k)=\int_{0}^{\infty} d x e^{-i k x} \mu(x)
$$

and

$$
P_{n}(z)=\int_{0}^{\infty} d t e^{-z t} \tilde{P}_{n}(t)
$$

First we need the waiting-time distribution $\tilde{h}(t)$ for the first jump of the light particle, which has been discussed by Tunaley. ${ }^{(24)}$ In equilibrium the initial time has constant probability to be anywhere between two subsequent jumps, and Feller ${ }^{(25)}$ has shown that

$$
\tilde{h}(t)=v \int_{t}^{\infty} d t^{\prime} \tilde{\psi}\left(t^{\prime}\right)
$$

or in Laplace transformed language

$$
h(z)=(\nu / z)[1-\psi(z)]
$$

where $\nu^{-1}=\int_{0}^{\infty} d t t \tilde{\psi}(t)$ is the average waiting time. Similarly, the probability $\tilde{s}(t)$ that the light particle, after arriving at a scatterer at time zero, does not jump within a time $t$ is given by

$$
\tilde{s}(t)=\int_{t}^{\infty} d t^{\prime} \tilde{\psi}\left(t^{\prime}\right)
$$

or

$$
s(z)=(1 / z)[1-\psi(z)]
$$

The probability $\tilde{s}_{0}(t)$ that a light particle remains at the same scatterer in the time interval from 0 to $t$ is

$$
\tilde{s}_{0}(t)=\int_{t}^{\infty} d t^{\prime} \tilde{h}\left(t^{\prime}\right)
$$


or

$$
s_{0}(z)=(1 / z)[1-h(z)]
$$

\subsection{Probabilities of Return and Site Occupancy}

Suppose the light particle jumps from scatterer 0 to a neighboring scatterer, say 1 , at the initial time $t=0$, then the Laplace transform $X(z)$ of the probability density for a first return to scatterer 0 satisfies the equation

$$
X(z)=\frac{1}{2} \psi(z) \sum_{n=0}^{\infty}\left[\frac{1}{2} \psi(z) X(z)\right]^{n}=\frac{\psi(z)}{2-\psi(z) X(z)}
$$

The $n$th term in this series contains the contributions from those processes where the light particle returns precisely $n$ times to scatterer 1 (from the right) before jumping back to scatterer 0. From (4.9) $X$ may be solved immediately, with the result

$$
X(z)=\frac{1}{\psi(z)}\left\{1-\left[1-\psi^{2}(z)\right]^{1 / 2}\right\}
$$

It follows then that the Laplace transform $R(z)$ of the unconditional probability density to return to scatterer 0 after an initial jump at $t=0$ is given as

$$
R(z)=\sum_{n=1}^{\infty} X^{n} \psi^{n-1}(z)=\frac{X}{1-\psi(z) X}
$$

Combination of the previous results yields for the Laplace transform of the probability density to end up at scatterer 0

$$
P_{0}(z)=s_{0}(z)+h(z) R(z) s(z)
$$

Similarly the Laplace transform $P_{n}(z)$ of the probability density to end up on scatterer $n$ at time $t$ is found as

$$
\begin{aligned}
P_{n}(z)= & P_{-n}(z)=\left(\frac{\frac{1}{2} h}{1-\frac{1}{2} \psi X}\right)\left(\frac{\frac{1}{2} \psi}{1-\frac{1}{2} \psi X}\right)^{|n|-1} s \\
& +h R\left(\frac{\frac{1}{2} \psi}{1-\frac{1}{2} \psi X}\right)^{|n|} s \\
= & \frac{h(z)}{\psi(z)[1-\psi(z) X(z)]}\left[\frac{\psi(z)}{2-\psi(z) X(z)}\right]^{|n|} s(z)
\end{aligned}
$$

where the terms account for all possibilities without [first term in (4.13a)] or with [second term in (4.13b)] intermediate returns to the origin; the factor 
$h(2-\psi X)^{-1}$ in (4.13a) accounts for the first jump from the origin to scatterer $k= \pm 1$, and all possible subsequent returns to $k$, avoiding the origin, whereas $\psi(2-\psi X)^{-1}$ accounts for any subsequent jump from scatterer $(k-1)$ to $k$ [if $k>0$, otherwise from $-(k-1)$ to $-k$ ] and all possible subsequent returns to $\pm k$ avoiding $\pm(k-1)$, where $k$ runs from 1 to $n$; the factor $h R$ in the second term of (4.13a) accounts for all possible returns to scatterer 0 . Finally, one has to multiply both terms in (4.13a) by a factor $s(z)$, as given in (4.7b).

\subsection{Green's Function and Time Correlation Functions}

Finally $G(k, z)$ is obtained from (4.3), (4.12), and (4.13) as

$$
\begin{aligned}
G(k, z) & =s_{0}+\frac{h X s}{1-\psi X}+\frac{h s}{\psi(1-\psi X)}\left[\sum_{n=1}^{\infty}\left(\frac{\psi M}{2-\psi X}\right)^{n}+\text { c.c. }\right] \\
& =s_{0}+\frac{h s}{1-\psi X}\left[X+\frac{M}{2-\psi M-\psi X}+\frac{M^{*}}{2-\psi M^{*}-\psi X}\right]
\end{aligned}
$$

where c.c. stands for the complex conjugate of the first term between square brackets. Hence we have expressed $G(k, z)$ in terms of the given functions $\psi(z)$ and $M(k)$.

In order to calculate correlation functions it is convenient to rewrite (4.14) introducing the quantities

$$
\begin{aligned}
& A(k)=M(k)+M(-k)-M(k) M(-k)-1 \\
& B(k)=M(k) M(-k)-1
\end{aligned}
$$

which, in the limit $k \rightarrow 0$, satisfy the equalities

$$
\lim _{k \rightarrow 0} A(k) / k^{2}=-l^{2}, \quad \lim _{k \rightarrow 0} B(k) / k^{2}=-\Delta^{2}
$$

After some algebraic rearrangements, employing (4.10), the result is

$$
G(k, z)=\frac{1}{z}+\frac{\frac{1}{2} h \alpha}{z(1-\psi)}
$$

with

$$
\begin{aligned}
\alpha(k, z)=\{A(k) & \left.+\left(\frac{1-\psi}{1+\psi}\right)^{1 / 2} B(k)\right\} /\left\{1-\frac{\frac{1}{2} \psi}{1-\psi} A(k)\right. \\
& \left.+\frac{1}{2}\left[1-\left(\frac{1+\psi}{1-\psi}\right)^{1 / 2}\right] B(k)\right\}
\end{aligned}
$$

To test the conjecture of Alley and Alder we have to calculate $\hat{U}(k, z)$ from 
(1.1b), which results in

$$
\hat{U}(k, z)=-\frac{z}{k^{2}} \frac{\frac{1}{2} h \alpha}{1-\psi} /\left(1+\frac{\frac{1}{2} h \alpha}{1-\psi}\right)
$$

Since we want to expand $\hat{U}(k, z)$ according to (1.6) up to $O\left(k^{2}\right)$ included, we have to consider the small- $k$ behavior of $M(k)$. Formally one has from (4.4)

$$
M(k)=\sum_{n=0}^{\infty} \frac{(-i k)^{n}}{n !}\left\langle x^{n}\right\rangle
$$

For our purposes it is sufficient that all moments up to $\left\langle x^{4}\right\rangle$ exist. In Eq. (4.6) the existence of an average waiting time $\nu^{-1}$ was already assumed; hence $\psi(z)$ may be expanded as $\psi(z)=1-z v^{-1}+o(z)$ for $z$ tending to zero. After some algebra we find for the velocity autocorrelation function $\phi_{2}(t)$ in $(1.7)$,

$$
\begin{aligned}
\hat{U}_{0}(z) & =\frac{z h(z)}{2[1-\psi(z)]}\left[l^{2}+\Delta^{2}\left(\frac{1-\psi(z)}{1+\psi(z)}\right)^{1 / 2}\right] \\
& =D+\frac{1}{2} \nu \Delta^{2}\left[\frac{1-\psi(z)}{1+\psi(z)}\right]^{1 / 2}
\end{aligned}
$$

In (4.20b) we have used Eq. (1.6) and introduced the diffusion coefficient $D=\hat{U}_{0}(0)=\frac{1}{2} \nu l^{2}$. The explicit form for $\hat{U}_{2}(z)$ is more complicated, and will not be given explicitly, since only its small- $z$ behavior will be needed. For the small- $z$ behavior we deduce

$$
\begin{aligned}
& \hat{U}_{0}(z) \simeq D+\frac{1}{2} \Delta^{2}(z v / 2)^{1 / 2} \\
& \hat{U}_{2}(z) \simeq \frac{1}{2} D \Delta^{2}(\nu / 2 z)^{1 / 2}
\end{aligned}
$$

The "velocity" correlation function $\phi_{2}(t)$, which should be interpreted as the second derivative of the mean square displacement, has, according to (4.21), a long-time tail ${ }^{(19,20)}$

$$
\phi_{2}(t) \simeq-\frac{\Delta^{2}}{4}\left(\frac{y}{2 \pi}\right)^{1 / 2} \frac{1}{t^{3 / 2}}
$$

which is in general agreement with the prediction for the deterministic Lorentz models. The coefficient $\Delta^{2}=\left\langle x^{2}\right\rangle-\langle x\rangle^{2}$ represents the fluctuations in the interval length, which corresponds to density fluctuations, and $\langle x\rangle=l=n^{-1}$. If one takes for these fluctuations a Poisson distribution $\mu(x)=l^{-1} \exp (-x / l)$, as is correct for a dilute gas, one finds from (4.23)

$$
\phi_{2}(t) \simeq-\frac{1}{4 n}\left(\frac{D}{\pi}\right)^{1 / 2} \frac{1}{t^{3 / 2}}
$$


in agreement with Eq. (3.9), for $d=1$. According to Eq. (4.22) the Burnett correlation function behaves for long times as

$$
\phi_{4}(t) \simeq \frac{1}{2} D \Delta^{2}(\nu / 2 \pi t)^{1 / 2}
$$

so that the conjecture of Alley and Alder is not satisfied for this model. The modified Burnett coefficient, $D_{4}(t) \sim D \Delta^{2}(v t / 2 \pi)^{1 / 2}$, as well as the ordinary one, $B(t) \simeq \frac{3}{2} D \Delta^{2}(v t / 2 \pi)^{1 / 2}$, diverge for long times.

\subsection{Periodic Lattice, Montroll-Weiss Model}

For the existence of a long-time tail in the velocity autocorrelation function $\phi_{2}(t)$ and for the even stronger tail in $\phi_{4}(t)$ it is very essential that there exist fluctuations in the density of scatterers. For a periodic lattice no tail exists. This can be seen very simply in the case of a sharp distribution $\mu(x)=\delta(x-l)$ for the interval length. In that case the coefficient of the tail, proportional to the variance $\Delta^{2}$, vanishes. Likewise the quantity $B(k)$, defined in (4.15), becomes equal to zero for all $k$. As a consequence of this $\hat{U}_{0}(z)$ becomes entirely frequency independent, irrespective of the form of $\psi(z)$, as was first noted by Tunaley ${ }^{(24)}$ and as can be seen immediately from (4.20). This implies that $\phi_{2}(t)$ has no long-time tail.

The special case of a sharp interval distribution for the waiting-time Lorentz model is also a special case of the Montroll-Weiss random walk with a waiting time, ${ }^{(23)}$ when the probability distribution $p(x)$ for a step size is sharp, i.e., $p(x)=\delta(x-l)$. Tunaley treats the general MontrollWeiss model with a waiting-time distribution $\tilde{h}(t)$ for the first jump that is chosen independent of the waiting-time distribution $\tilde{\psi}(t)$ for all following jumps. He then shows that the Laplace transform $\hat{C}_{0}(z)$ of the velocity autocorrelation function is of the form

$$
\hat{C}_{0}(z)=\hat{U}_{0}(z) \sim \frac{z h(z)}{1-\psi(z)}
$$

with a proportionality constant independent of $z$ in complete agreement with (4.20a) with $\Delta=0$. If one chooses $\tilde{h}(t)$ to be equal to $\tilde{\psi}(t)$ (this is the only case considered by Montroll and Weiss in Ref. 23) and if in addition one assumes that $\tilde{\psi}(t)$ has a long-time tail proportional to $t^{-\alpha}$, with $\alpha>2$, so that the average waiting time exists, then $\hat{U}_{2}(z)$ contains a leading nonanalyticity $\sim z^{\alpha-1}$ for $z$ tending to 0 . This corresponds to a $t^{-\alpha}$ long-time tail in the velocity autocorrelation function, and it is easily shown that in this case all functions $\phi_{2 n}(t)$, as introduced in (1.5), have a similar long-time tail proportional to $t^{-\alpha}$. It was this observation, besides the evidence from molecular dynamics results, that motivated Alley and Alder's conjecture. ${ }^{(17,18)}$ However, in equilibrium the assumption that $\tilde{h}(t)$ equals 
$\tilde{\psi}(t)$ is not justified, and instead these two waiting-time distributions are related to each other through (4.6). Substitution of (4.6b) into (4.26) immediately leads to Tunaley's result of a $\hat{U}_{0}$ that is independent of $z$, irrespective of the waiting-time distribution. This implies that for MontrollWeiss waiting-time models in equilibrium the conjecture of Alley and Alder is not satisfied. Hence, if this conjecture is satisfied for deterministic and stochastic Lorentz model in equilibrium, as seems to be the case, this cannot be explained on the basis of a reduction of those models to some effective Montroll-Weiss waiting-time model.

\section{DISCUSSION}

\subsection{Model Dependence of Results}

We have investigated four different types of models to test the conjecture of Alder and Alley that the four-point correlation function involved in $\phi_{4}(t)$ decays at large times sufficiently fast that it can be expressed in the two-point velocity correlation function $\phi_{2}(t)$, and consequently a modified Burnett coefficient $D_{4}$ or $D_{4 h}$ [see Eqs. (1.17)-(1.18)] would exist in systems where the diffusion coefficient itself is finite:

(i) For the three-dimensional fluid we have disproved the conjecture as expressed by (1.18) on the basis of a low-density kinetic theory or the mode-coupling theory, valid for general densities. Both theories have given a satisfactory explanation of the existence of the long-time tails themselves.

(ii), (iii) For the $d$-dimensional deterministic Lorentz model $(d \geqslant 2)$ and for the stochastic Lorentz model $(d \geqslant 1)$, as introduced in Section 1, the conjecture has been confirmed in the low-density limit by means of kinetic theory. In addition, for the one-dimensional stochastic Lorentz model a rigorous proof of the conjecture is available ${ }^{(19,20)}$ for general "density" (in this case the density is replaced by the probability $p$ for reflection of the light particle upon collision with a scatterer ${ }^{(22)}$ ) and for a general distribution of the length of the intervals between neighboring scatterers.

The kinetic theory methods employed suggest more generally that for long times and low densities $\phi_{2 n}(t) \sim \phi_{2}(t)$, in agreement with the extended $(n>2)$ conjecture of Alley and Alder, ${ }^{(17,18)}$ as discussed in Section 1.4. The reason is that the so-called ring diagrams for $\hat{U}(\mathbf{k}, z)$ or $\hat{C}(\mathbf{k}, z)$ depend only on the external $\mathbf{k}$ through $\exp (i \mathbf{k} \cdot \hat{\boldsymbol{\sigma}} \boldsymbol{\sigma})$, appearing in the binary collision operators $\overline{\mathbf{T}}_{\mathbf{k}}$ and $\mathbf{T}_{k}$. This $\mathbf{k}$ dependence is regular for small $k$ and is neglected in our low-density kinetic theory. That the internal propagators inside the rings do not depend on $\mathbf{k}$ - and that, consequently, the sum of the diagrams of class $\mathrm{V}$, as calculated in the Appendix, vanishes-is a direct consequence of the fact that the first and last collisions in a ring event 
occur with the same scatterer, hence almost at the same position. In the fluid, however, the propagators $\boldsymbol{\Gamma}_{\mathbf{k}}(z)$ (see Section 3), appearing inside the ring diagrams, do depend on the external $\mathbf{k}$. This is the reason why the small- $z$ behavior of $\hat{U}_{2 n}(z)$ becomes more strongly divergent in the limit $z \rightarrow 0$ with increasing $n$. In the higher-order "self-energy" diagrams the internal propagators depend on the external $\mathbf{k}$ also in the Lorentz models. Therefore, without doing further calculations, one cannot conclude from kinetic theory whether or not the conjecture of Alley and Alder remains valid in Lorentz models at higher densities. On the other hand, for the one-dimensional stochastic Lorentz models of Section 3 the conjecture relating $\phi_{4}(t)$ to $\phi_{2}(t)$ is confirmed by an exact solution for general densities, ${ }^{(19,20)}$ but cannot be explained on the basis of an effective reduction to a Montroll-Weiss waiting-time model, in which the velocity autocorrelation function in equilibrium has no long-time tail, as can be concluded from Tunaley's work. ${ }^{(24)}$

\subsection{Molecular Dynamics Data}

Our last point is a comparison of the various new predictions with the results obtained from computer simulations. First we consider the long-time tails occurring in the correlation functions $\phi_{2}(t)$ and $B(t)$, as introduced in (3.9) and (3.16), respectively. Here we follow the presentation of Alley and Alder. Introducing a dimensionless time $s=\nu t$ and a dimensionless density $n^{*}=n \sigma^{2}$ (where $\sigma$ is the radius of the scatterers) Alley ${ }^{(18)}$ has presented these functions for the two-dimensional Lorentz gas at large times as ${ }^{5}$

$$
\phi_{2}(t) / \phi_{2}(0) \simeq-\alpha_{D} s^{-\beta_{D}}, \quad D_{0}^{-2} d B(t) / d t \simeq \alpha_{B} s^{-\beta_{B}}
$$

Here $\phi_{2}(0)=\frac{1}{2} v^{2}$; the Boltzmann value of the diffusion coefficient, given by (3.4), is indicated in this discussion as $D_{0}$ in order to distinguish it from the actual diffusion coefficient $D$ and $\beta_{D}=\beta_{B}+1=2$. We further have the low-density predictions

$$
\alpha_{D}=\frac{n^{*}}{\pi}, \quad \alpha_{B}=\frac{4 n^{*}}{3 \pi}
$$

For the velocity correlation function comparisons with molecular dynamics results have been made by Bruin, ${ }^{(14)}$ Alder and Alley, ${ }^{(16-18)}$ and Lewis and Tjon ${ }^{(15)}$; for the super Burnett coefficient the comparison is new (see Table I). Only at low densities $\left(n^{*} \leqslant 0,10\right)$ are the exponents $\beta_{D}$ and $\beta_{B}$ found in the computer simulations in agreement with the theoretical predictions. The coefficients $\alpha_{D}$ and $\alpha_{B}$ are far away from the low-density kinetic theory

${ }^{5}$ Unfortunately the caption of Table I of Ref. 16 suggests that $\alpha_{B}$ is the coefficient of the long-time tail of $\left\{d^{2}(t B) d t^{2}\right\} / D_{0}^{2}$ instead of $\{d B / d t\} / D_{0}^{2}$. 
Table I. Tail Coefficients for the Two-Dimensional Deterministic Lorentz Gas from Molecular Dynamics Calculations

\begin{tabular}{lccc}
\hline \multicolumn{1}{c}{$n^{* a}$} & $\alpha_{D} / n^{*}$ & $\alpha_{B} / n^{*}$ & $\frac{3}{4} \frac{D_{0}}{D} \frac{\alpha_{B}}{\alpha_{D}}$ \\
\hline 0 & $1 / \pi \approx 0.3$ & $4 / 3 \pi \approx 0.4$ & 1 \\
$0.03(0)^{b}$ & $0.87 \pm 0.16$ & $1.3 \pm 0.7$ & $1.3 \pm 0.9$ \\
$0.05(0)^{b}$ & $1.2 \pm 0.1$ & $1.6 \pm 0.4$ & $1.2 \pm 0.4$ \\
$0.05(N)^{c}$ & $0.75 \pm 0.03$ & & \\
$0.10(0)^{b}$ & $2.0 \pm 0.5$ & $1.6 \pm 0.2$ & $0.9 \pm 0.4$ \\
\hline
\end{tabular}

${ }^{a} O$, overlapping disks; $N$, nonoverlapping disks. The values for $n^{*} \rightarrow 0$ are the kinetic theory results.

${ }^{b}$ Reference 18.

${ }^{c}$ Reference 15.

results. However, a linear extrapolation through the three available values for $\alpha_{D}$ at the reduced densities $n^{*}=0.03,0.05$, and 0.10 is perfectly in agreement with the theoretical limit for $n^{*} \rightarrow 0$, as was demonstrated by Alley and Alder. ${ }^{(18)}$ For $\alpha_{B}$ a similar extrapolation through two data points is not inconsistent with the theoretical value in the limit $n^{*} \rightarrow 0$, but the uncertainties in these points are so large that no stronger conclusions can be drawn. It should be mentioned, further, that the conjecture of Alley and Alder, if it is valid, immediately leads to a relation between $\alpha_{B}$ and $\alpha_{D}$ that can be obtained by identifying the dominant singularities on both sides of Eq. (1.10) under the assumption that $D_{4}$ in (1.17) is finite, i.e.,

$$
\alpha_{B}=\frac{4}{3}\left(D / D_{0}\right) \alpha_{D}
$$

where $D$ is the actual diffusion coefficient and $D_{0}$ is its value as calculated from the Boltzmann equation. In the last column of Table I we list the values of $\frac{3}{4}\left(D_{0} / D\right)\left(\alpha_{B} / \alpha_{D}\right)$ as obtained from Alley and Alder's molecular dynamics results $^{(18)}$ for $\alpha_{B}, \alpha_{D}$, and $D$. Given the error bounds, these values are always close to unity indeed.

It is remarkable that higher-density corrections to the low-density kinetic theory become so important already at fairly low densities, such as $n^{*}=0.03$. The existence of strong density effects also becomes clear from the large difference between the results for $\alpha_{D}$ in the overlapping and the nonoverlapping disk system (see Table I).

On the basis of kinetic theory we showed that for low densities the modified Burnett coefficient $D_{4}$ is finite for the two-dimensional Lorentz gas, in agreement with the conjecture of Alley and Alder. It would be worthwhile to calculate analytically all ring contributions (diagrams I, II, ..., V in Section 3) to lowest nonvanishing order in the density, in order to obtain an estimate for the leading higher-density corrections to the 
Table II. Diffusion and Burnett Coefficients for the Two-Dimensional Overlapping Lorentz Gas

\begin{tabular}{llll}
\hline \multicolumn{1}{r}{$n^{*}$} & \multicolumn{1}{c}{$D / D_{0}$} & $D_{4} v / D_{0}^{2}$ & $D_{4} / D \lambda^{2}$ \\
\hline 0 & 1 & 0.47 & 0.18 \\
0.02 & $0.92 \pm 0.02$ & $0.57 \pm 0.04$ & $0.23 \pm 0.02$ \\
0.05 & $0.81 \pm 0.01$ & $0.50 \pm 0.04$ & $0.23 \pm 0.02$ \\
\hline
\end{tabular}

Boltzmann value of $D_{4}$, as given in (3.17), but thus far this calculation has not been undertaken. In Table II we compare the Boltzmann value of $D_{4}$ with molecular dynamics values given by Alley and Alder for the twodimensional Lorentz gas. In this table $\lambda=v / \nu$ is the mean free path and $D$ is the value of the diffusion coefficient as obtained from molecular dynamics [18] again. From (3.17) one obtains the Boltzmann values (listed in Table II as $n^{*}=0$ values)

$$
\frac{D_{4} \nu}{D_{0}^{2}}=\frac{15}{32}, \quad \frac{D_{4}}{D \lambda^{2}}=\frac{45}{256}
$$

One sees that even at these low densities the diffusion coefficient $D$ and the modified Burnett coefficient $D_{4}$ contain appreciable contributions from higher-density corrections to the Boltzmann equation.

\section{ACKNOWLEDGMENTS}

It is a great pleasure to thank Dr. W. W. Wood and Dr. J. Erpenbeck for suggesting the problem, for making the unpublished Fig. 1 available to us, and for their collaboration on the results of the three-dimensional fluid. In addition one of us (M.H.E.) wants to thank them for their hospitality during a visit at the Los Alamos Scientific Laboratories in August, 1979, where this work was started. We are also indebted to Mr. J. Koenen for his help in calculating the super Burnett coefficient for the two-dimensional Lorentz model. One of us (M.H.E.) wants to thank Dr. B. J. Alder for several stimulating discussions and for sending him Ref. 18.

\section{APPENDIX}

From Eqs. (3.14) and (3.15) we have

$$
\left\langle\hat{v}_{x} B_{x x}(z) \hat{v}_{x}\right\rangle=\int^{\prime} \frac{d \mathbf{k}}{(2 \pi)^{d}}\left\langle\hat{v}_{x} \boldsymbol{\Gamma}_{\mathbf{k}}^{B}(z) \hat{v}_{x} \boldsymbol{\Gamma}_{\mathbf{k}}^{B}(z) \hat{v}_{x} \boldsymbol{\Gamma}_{\mathbf{k}}^{B}(z) \hat{v}_{x}\right\rangle
$$


As a preparation we obtain from (3.8) equalities valid to lowest order in $k$ :

$$
\begin{aligned}
\left\langle\psi_{\mathbf{k}} \hat{v}_{x} \psi_{\mathbf{k}}\right\rangle & =2\left\langle\hat{v}_{x} \psi_{\mathbf{k}}\right\rangle=2 i k_{x} D / v \\
\left\langle\psi_{\mathbf{k}} \hat{v}_{x} Q_{0}\left\{-n \mathbf{T}_{0}\right\}^{-1} Q_{0} \hat{v}_{x} \psi_{\mathbf{k}}\right\rangle & =\left\langle\psi_{\mathbf{k}} \hat{v}_{x} Q_{0}\left\{-n \mathbf{T}_{0}\right\}^{-1} Q_{0} \hat{v}_{x}\right\rangle \\
& =\left\langle\hat{v}_{x}\left(-n \mathbf{T}_{0}\right)^{-1} \hat{v}_{x}\right\rangle=D / v^{2}
\end{aligned}
$$

Then we have the following contributions using (3.8)

$$
\begin{aligned}
(s s s)= & \int^{\prime} \frac{d \mathbf{k}}{(2 \pi)^{d}} \frac{1}{\left(z+D k^{2}\right)^{3}}\left\langle\hat{v}_{x} \psi_{\mathbf{k}}\right\rangle^{2}\left\langle\psi_{\mathbf{k}} \hat{v}_{x} \psi_{\mathbf{k}}\right\rangle^{2} \\
= & \frac{4 D^{4}}{v^{4}} \int^{\prime} \frac{d k}{(2 \pi)^{d}} \frac{k_{x}^{4}}{\left(z+D k^{2}\right)^{3}} \\
(f s s)+(s s f)= & 2 \int^{\prime} \frac{d \mathbf{k}}{(2 \pi)^{d}} \frac{1}{\left(z+D k^{2}\right)^{2}}\left\langle\hat{v}_{x} \psi_{\mathbf{k}}\right\rangle\left\langle\psi_{\mathbf{k}} \hat{v}_{x} \psi_{\mathbf{k}}\right\rangle \\
& \times\left\langle\psi_{\mathbf{k}} \hat{v}_{x} Q_{0}\left(-n \mathbf{T}_{0}\right)^{-1} Q_{0} \hat{v}_{x}\right\rangle \\
= & -\frac{4 D^{3}}{v^{3}} \int^{\prime} \frac{d \mathbf{k}}{(2 \pi)^{d}} \frac{k_{x}^{2}}{\left(z+D k^{2}\right)^{2}} \\
(s f s)= & \int^{\prime} \frac{d \mathbf{k}}{(2 \pi)^{d}} \frac{1}{\left(z+D k^{2}\right)^{2}}\left\langle\hat{v}_{x} \psi_{\mathbf{k}}\right\rangle^{2}\left\langle\psi_{\mathbf{k}} \hat{v}_{x} Q_{0}\left(-n \mathbf{T}_{0}\right)^{-1} Q_{0} \hat{v}_{x} \psi_{\mathbf{k}}\right\rangle \\
= & -\frac{D^{3}}{v^{4}} \int^{\prime} \frac{d \mathbf{k}}{(2 \pi)^{d}} \frac{k_{x}^{2}}{\left(z+D k^{2}\right)^{2}} \\
(f s f)= & \int^{\prime} \frac{d \mathbf{k}}{(2 \pi)^{d}}\left(\frac{1}{z+D k^{2}}\right)\left\langle\hat{v}_{x} Q_{0}\left(-n \mathbf{T}_{0}\right)^{-1} Q_{0} \hat{v}_{x} \psi_{\mathbf{k}}\right\rangle^{2} \\
= & \frac{D^{2}}{v^{4}} \int^{\prime} \frac{d \mathbf{k}}{(2 \pi)^{d}}\left(\frac{1}{z+D k^{2}}\right)
\end{aligned}
$$

Next we carry out the angular $\mathbf{k}$ integrations, using $\left\langle\hat{\boldsymbol{v}}_{x}^{2}\right\rangle=d^{-1}$ and $\left\langle\hat{v}_{x}^{4}\right\rangle=3[d(d+2)]^{-1}$, sum the terms (A.4)-(A.7), and take the inverse Laplace transform to find for the dominant long-time behavior

$$
\left\langle\hat{v}_{x} B_{x x}(t) \hat{v}_{x}\right\rangle \simeq \frac{D^{2}}{v^{4}}\left(\frac{1}{4 \pi^{2} D t}\right)^{d / 2} \int d \mathbf{x} e^{-x^{2}}\left[1-\frac{5}{d} x^{2}+\frac{6}{d(d+2)} x^{4}\right]=0
$$


where we introduced the new variables $\mathbf{x}=(D t)^{1 / 2} \mathbf{k}$. Although it looks as if the function in (A.8) has a stronger tail $\left(\sim t^{-d / 2}\right)$ than the correlation function $\phi_{2}(t) \sim t^{-d / 2-1}$, its coefficient vanishes, and the tail of $C_{2}^{(\mathrm{I})}(t)$ in (3.14) is of the same order in $t$ as the tail of $\phi_{2}(t)$.

\section{REFERENCES}

1. R. Zwanzig, Phys. Rev. 124:983 (1961); H. Mori, Prog. Theor. Phys. 33:423 (1965); J. R. Dorfman and M. H. Ernst, J. Stat. Phys. 12:311 (1975).

2. J. A. McLennan, Phys. Rev. A 8:1479 (1973).

3. I. M. de Schepper, H. van Beijeren, and M. H. Ernst, Physica 75:1 (1974).

4. I. M. de Schepper and M. H. Ernst, Physica 98A:189 (1979); I. M. de Schepper, Generalized Hydrodynamics for the Diffusion Process, doctoral thesis, University of Nijmegen (1975).

5. B. J. Alder and T. E. Wainwright, Phys. Rev. $A$ 1:18 (1970).

6. M. H. Ernst, E. H. Hauge, and J. M. J. van Leeuwen, Phys. Rev. Lett. 25: 1254 (1970); J. R. Dorfman and E. G. D. Cohen, Phys. Rev. Lett. 25:1257 (1970).

7. M. H. Ernst and A. Weyland, Phys. Lett. 34A:39 (1971).

8. E. H. Hauge, What Can One Learn from Lorentz Models? In Lecture Notes in Physics Vol. 31, G. Kirczenow and J. Marro, eds. (Springer, Berlin, 1974).

9. P. Résibois and Y. Pomeau, Phys. Rep. 19C:63 (1975).

10. T. Keyes and I. Oppenheim, Physica, 70A:100 (1973); J. W. Dufty and J. A. McLennan, Phys. Rev. A 9:1266 (1974); D. Bedeaux and P. Mazur, Physica 73A:431 (1974).

11. W. W. Wood, in Fundamental Problems in Statistical Mechanics, Vol. 3, E. G. D. Cohen ed. (N.H.P.C., Amsterdam, 1975).

12. W. W. Wood and J. J. Erpenbeck, J. Stat. Phys., to appear.

13. J. M. J. van Leeuwen and A. Weyland, Physica 36:457 (1967); 38:35 (1968).

14. C. Bruin, Phys. Rev. Lett. 29:1670 (1972); Physica 72:261 (1974).

15. J. C. Lewis and J. A. Tjon, Phys. Lett. 66A:349 (1978).

16. B. J. Alder and W. E. Alley, J. Stat. Phys. 19:34l (1978). B. J. Alder, in Lecture Notes in Physics, Vol. 84, L. Garrido, P. Seglar, and P. J. Shepherd eds. (Springer, Berlin, 1978).

17. B. J. Alder and W. E. Alley, Phys. Rey. Lett. 43:653 (1979).

18. W. E. Alley, Studies in Molecular Dynamics of the Friction Coefficient and the Lorentz Gas, Ph.D. thesis, University of California/Livermore (1979).

19. H. van Beijeren, Rev. Mod. Phys, to be published.

20. H. van Beijeren and $H$. Spohn, to be published.

21. A. Weyland, J. Math. Phys. 15:1942 (1974).

22. P. Grassberger, Physica, 103A:558 (1980).

23. E. W. Montroll and G. H. Weiss, J. Math. Phys. 6:167 (1965).

24. J. K. E. Tunaley, Phys. Rev. Lett. 33:1037 (1974).

25. W. Feller, An Introduction to Probability Theory and its Applications, Vol. 2, 2nd ed. (Wiley, New York, 1971). 